\title{
A Compact SOI-Integrated Multiwavelength Laser Source Based on Cascaded InP Microdisks
}

\author{
Joris Van Campenhout, Member, IEEE, Liu Liu, Pedro Rojo Romeo, Dries Van Thourhout, Member, IEEE, \\ Christian Seassal, Philippe Regreny, Lea Di Cioccio, Jean-Marc Fedeli, and Roel Baets, Fellow, IEEE
}

\begin{abstract}
We report on the performance of a compact multiwavelength laser (MWL) source heterogeneously integrated with and coupled to a silicon-on-insulator (SOI) waveguide circuit. The MWL consists of four InP-based microdisk lasers, coupled to a common SOI wire waveguide. The microdisk lasers operate in continuous-wave regime at room temperature, with a threshold current around $0.9 \mathrm{~mA}$ and a waveguide-coupled slope efficiency of up to $8 \mu \mathrm{W} / \mathrm{mA}$, for a microdisk diameter of $7.5 \mu \mathrm{m}$. The output spectrum contains four laser peaks uniformly distributed within the free-spectral range of a single microdisk. While thermal crosstalk is negligible, laser peak output powers vary up to $8 \mathrm{~dB}$ for equal microdisk drive currents, as a result of loss due to coupling with higher order modes supported by the 1- $\mu \mathrm{m}$-thick microdisks. This nonuniformity could be eliminated by reducing the microdisk thickness.
\end{abstract}

Index Terms-Heterogeneous integration, microdisk laser, multiwavelength laser source, silicon photonics.

\section{INTRODUCTION}

$\mathbf{O}$ VER the last 15 years, microdisk lasers have attracted considerable attention for their potential as compact and low-threshold coherent light sources for densely integrated photonic circuits [1]-[3]. The functionality of such photonic circuits could be vastly expanded by integrating them with silicon-based complementary metal-oxide-semiconductor electronic circuits. Furthermore, exploiting the existing silicon processing infrastructure could prove to be a cost-effective route for the fabrication of these electronic-photonic integrated circuits. Recently, we demonstrated electrically injected laser operation for an InP-based microdisk laser integrated with and coupled to a nanophotonic silicon-on-insulator (SOI) wire waveguide [4]. Since the lasing wavelength of a microdisk laser is fixed through its diameter, they are attractive

Manuscript received April 1, 2008; revised May 9, 2008. This work was supported in part by the EU-funded FP7 ICT Project WADIMOS and in part by the Belgian IAP Network photonics@be. The work of J. Van Campenhout was supported by the Research Foundation-Flanders (FWO-Vlaanderen) through a doctoral fellowship.

J. Van Campenhout is with the IBM T. J. Watson Research Center, Yorktown Heights, NY 10598 USA.

L. Liu, D. Van Thourhout, and R. Baets are with the Photonics Research Group of the Department of Information Technology (INTEC), Ghent University-IMEC, 9000 Ghent, Belgium (e-mail: Dries.VanThourhout@intec.UGent. be).

P. Rojo Romeo, C. Seassal, and P. Regreny are with the Université de Lyon, Institut des Nanotechnologies de Lyon INL-UMR5270, CNRS, Ecole Centrale de Lyon, Ecully, F-69134, France.

L. Di Cioccio and J.-M. Fedeli are with the CEA-LETI Minatec, 38054 Grenoble, France.

Color versions of one or more of the figures in this letter are available online at http://ieeexplore.ieee.org.

Digital Object Identifier 10.1109/LPT.2008.926857

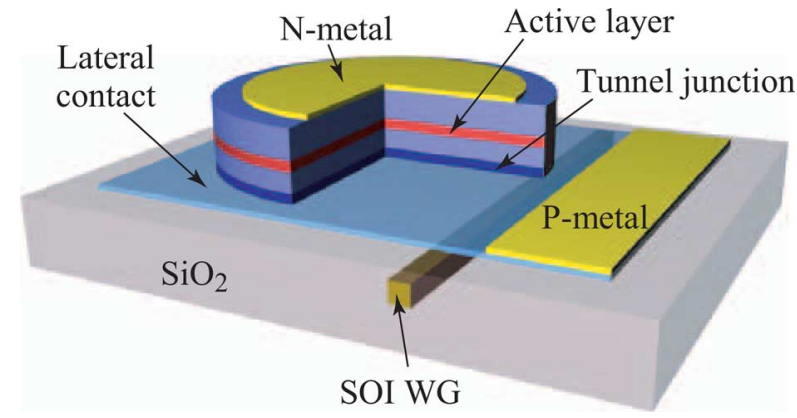

Fig. 1. Layout of a single SOI-integrated microdisk laser.

candidates as single-mode lasers for applications requiring wavelength-division multiplexing [5]. Here, we report on a compact, silicon-integrated multiwavelength laser (MWL) source based on an array of such waveguide-coupled microdisk lasers.

\section{SingLe LASER StRUCTURE}

The microdisk lasers are fabricated in a $1-\mu \mathrm{m}$-thick InP-based epitaxial layer structure, which was directly bonded on top of a planarized SOI waveguide layer stack. The n-type top contact is positioned over the center of the disk, whereas the p-type contact can be found on a thin lateral bottom contact layer (see Fig. 1). A tunnel junction was used to achieve low contact resistance to the p-type layer of the p-i-n structure, while maintaining a relatively low internal optical loss. The underlying SOI output waveguide $\left(500 \times 220 \mathrm{~nm}^{2}\right.$ in cross section) is aligned with the edge of the microdisk, in order to obtain maximum evanescent coupling between the waveguide mode and the microdisk's whisperinggallery mode. We refer to [4] for further details on the epitaxial layer structure, the laser layout, and the fabrication.

The typical light output-current-voltage $(L-I-V)$ data for a $7.5-\mu \mathrm{m}$ diameter microdisk laser is shown in Fig. 2 . The threshold current is $0.9 \mathrm{~mA}$ and the slope efficiency is about $8 \mu \mathrm{W} / \mathrm{mA}$. The maximum output power in the continuous-wave $(\mathrm{CW})$ regime is limited to $12 \mu \mathrm{W}$ due to early thermal roll-over. The oscillations in the $L-I$ curve are due to reflection feedback arising from the grating couplers, which were used for fiber-chip coupling [6]. The threshold voltage is as low as $1.15 \mathrm{~V}$. This lower voltage and higher threshold current as compared to the results presented in [4] are most likely due to higher tunnel-junction doping levels, resulting in a decreased tunnel-junction resistance but an increased internal loss [7]. Furthermore, the slope efficiency is relatively low, due to the thicker bonding layer thickness of $180 \mathrm{~nm}$ as compared to $130 \mathrm{~nm}$ in [4]. It should be noted that the laser efficiency 


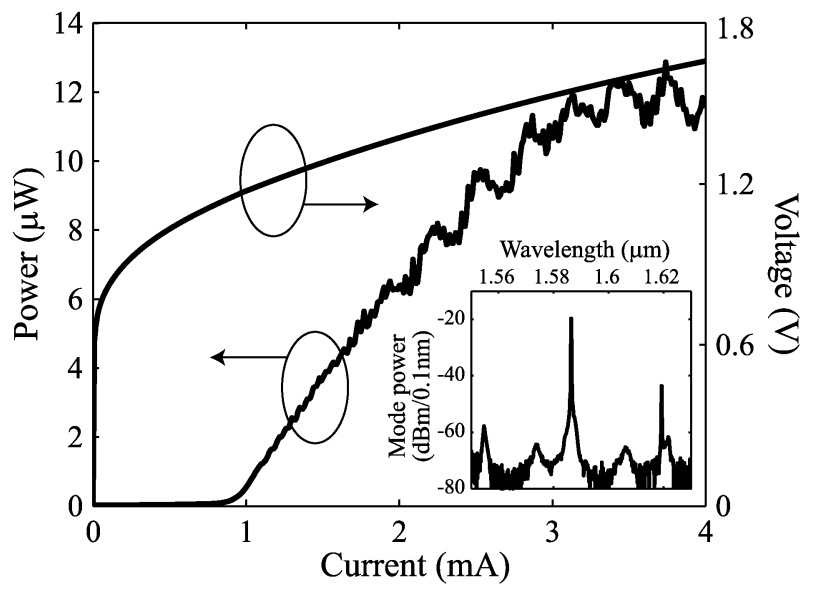

Fig. 2. Typical measured $L-I-V$ data for a single 7.5- $\mu \mathrm{m}$ diameter microdisk laser. The inset shows the lasing spectrum at $4.0 \mathrm{~mA}$.

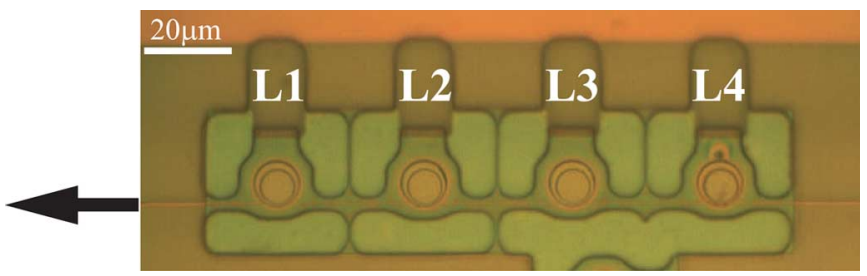

Fig. 3. Microscope image of the MWL source (prior to metallization) consisting of four microdisk lasers coupled to a common, underlying SOI wire. The arrow indicates the position of the monitor grating coupler.

and output power could be improved more than tenfold by optimizing the microdisk radius, bonding layer thickness, and tunnel-junction doping [7].

\section{Multiwavelength Laser Structure}

The MWL structure consists of four microdisk lasers coupled to a common SOI wire waveguide, as illustrated in Fig. 3. The nominal microdisk diameters were 7.632, 7.588, 7.544, and $7.5 \mu \mathrm{m}$ for lasers L1, L2, L3, and L4, respectively, such that the laser peaks are uniformly spread within the free-spectral range $\Delta \lambda_{\mathrm{FSR}}$ of a single microdisk. The output SOI waveguide is terminated at both ends by a grating coupler. The MWL output spectrum measured at the leftmost grating coupler-normalized for the grating coupler spectrum-with all lasers biased at $3.5 \mathrm{~mA}$ is shown in Fig. 4. It can be seen that the laser peaks of lasers L1, L4, and L3 are indeed almost uniformly distributed within the free-spectral range $(32 \mathrm{~nm})$ of laser $\mathrm{L} 2$, which is almost lasing in two modes. The lasing wavelengths of nominally identical microdisk lasers on the same chip and under identical driving conditions varied by less than $\pm 500 \mathrm{pm}$.

However, the laser peak belonging to the laser positioned the furthest away (L4) from the used grating coupler is about $8 \mathrm{~dB}$ weaker than that of the nearest one (L1). For the lasers positioned closer to the monitor grating coupler, we observe a gradual increase in output power. Therefore, we believe that a large fraction of this nonuniformity is attributed to coupling losses to the vertically higher order modes of the relatively thick

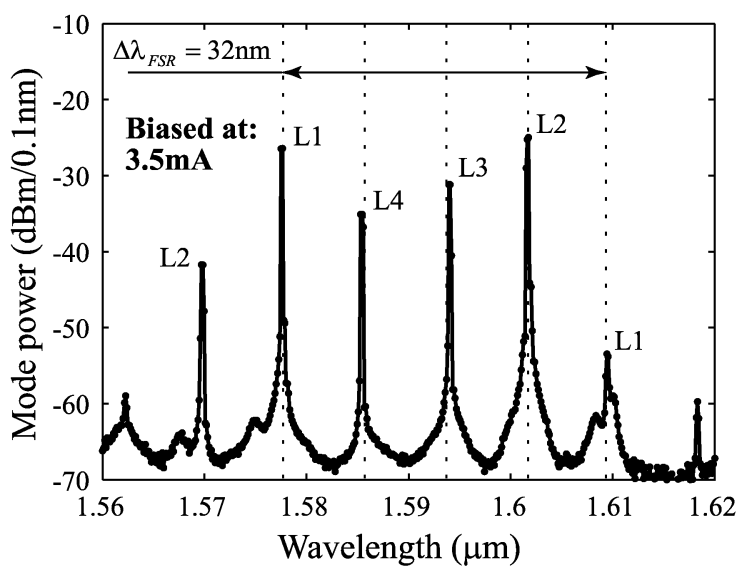

Fig. 4. Measured output spectrum for the MWL for a drive current of $3.5 \mathrm{~mA}$ for each of the four microdisk lasers. The dotted lines indicate the equally distributed wavelengths.

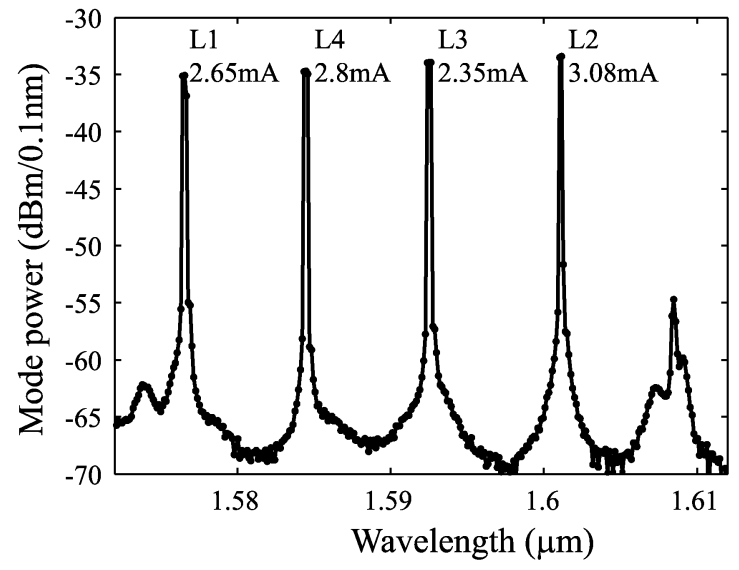

Fig. 5. Balanced output spectrum obtained by adjusting the individual microdisk drive currents.

microdisks $(1 \mu \mathrm{m})$, when the laser light passes under the neighboring disks on its way to the grating coupler. This assumption was verified by grating-to-grating transmission measurements and numerical simulations which revealed a much greater disk-waveguide coupling for these higher order modes as compared to the fundamental lasing mode. Further contributions to the nonuniformity arise from variations in fabrication and from spectral misalignment of the laser peak with the peak gain wavelength.

The MWL output spectrum can be made uniform be applying different bias currents to the microdisk lasers, as shown in Fig. 5. However, this approach might be difficult for MWLs with a large number of wavelength channels, as the nonuniformity scales directly with the number of microdisks. An alternative solution is to use a thinner InP-based epitaxial layer structure which is single-mode in the vertical direction, thereby effectively eliminating the coupling loss. Simulations indicate that for a single-mode thickness of $300 \mathrm{~nm}$, the nonuniformity could be suppressed to less than $0.1 \mathrm{~dB}$ per waveguide-disk crossing. However, for these thinner disks, the contribution of the absorption loss in the tunnel junction to the total internal loss grows rapidly and the doping levels should be lowered accordingly 

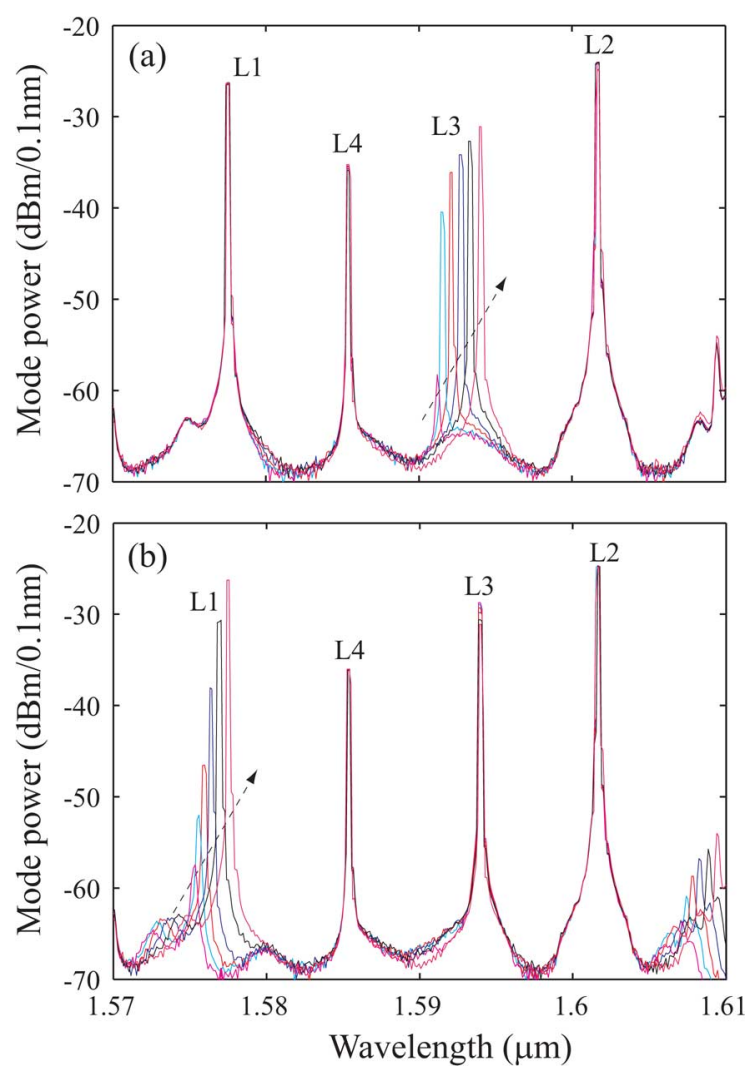

Fig. 6. Spectrum obtained with different bias currents of one microdisk laser (a) for L3 and (b) for L1. Along the arrow direction, the corresponding bias current increases from 1.0 to $3.5 \mathrm{~mA}$ with a step of $0.5 \mathrm{~mA}$. The other three microdisk lasers are all biased at $3.5 \mathrm{~mA}$.

to maintain the lasing ability, at the expense of an increased electrical device resistance [7]. While we have achieved lasing in tunnel-junction-based microdisk lasers as thin as $0.5 \mu \mathrm{m}$ [8], truly single-mode microdisks will require a different electrical injection scheme. A promising approach could be to inject the holes through a central, heavily p-type-doped post on top of the disk, which then acts as a low-resistance p-type contact, as was done in [9] for a photonic-crystal laser. Electrons would be injected through the lateral bottom contact layer.

The crosstalk of the present MWL was also evaluated by varying the bias current of one microdisk laser and recording the whole spectrum while keeping the drive conditions of the other lasers unchanged. An example of such a measurement is shown in Fig. 6. Due to the lack of a good thermal sink, the lasing wavelength of the corresponding microdisk shifted clearly when changing the drive current. The measured thermal resistance was about $6.4 \mathrm{~K} / \mathrm{mW}$, which is consistent with the theoretical modeling [8]. However, for the other three microdisk lasers, the lasing wavelengths remained unchanged (at least within the resolution limit of the spectrum analyzer, i.e., $1.0 \mathrm{pm}$ ), meaning that the thermal crosstalk is negligible, even though the distance between adjacent microdisks is only $33 \mu \mathrm{m}$ (see Fig. 3). Generally, the peak intensities of the other microdisk lasers are also stable, but in some cases they can vary by several decibels [e.g., L3 peak in Fig. 6(b)]. This is again due to the presence of high order modes in the microdisk. Besides the background coupling loss (as we discussed above), these high order modes will give extra resonant absorption losses at their resonant wavelengths. This is also confirmed by the transmission measurements, where we saw multiple resonant dips. For some combinations of microdisk drive currents, a lossy higher order mode of a disk positioned closer to the monitor grating coupler can get spectrally aligned with the lasing mode of another microdisk positioned further away from the grating coupler. In that case, the measured output power of the latter will be affected through this resonant coupling loss. This effect can also be eliminated by reducing the thickness of the microdisk lasers.

\section{CONCLUSION}

We have presented a compact silicon-integrated MWL source consisting of four InP-based microdisk lasers coupled to a common output SOI wire waveguide. The threshold current and voltage were $0.9 \mathrm{~mA}$ and $1.15 \mathrm{~V}$, respectively, with waveguide-coupled $\mathrm{CW}$ slope efficiencies up to $8 \mu \mathrm{W} / \mathrm{mA}$. The MWL output spectrum contained four laser peaks, uniformly distributed by design within the free-spectral range of a single microdisk. At equal drive currents, the peak power of the channels varied up to $8 \mathrm{~dB}$, due to loss caused by parasitic coupling to the higher order modes of the neighboring microdisks. The channel uniformity could be improved be reducing the microdisk thickness. Thermal crosstalk was negligible.

\section{REFERENCES}

[1] A. F. J. Levi, R. E. Slusher, S. L. Mccall, T. Tanbun-Ek, D. L. Coblentz, and S. J. Pearton, "Room temperature operation of microdisc lasers with submilliamp threshold current," Electron. Lett., vol. 28, no. 11, pp. 1010-1012, May 21, 1992.

[2] T. Baba, M. Fujita, A. Sakai, M. Kihara, and R. Watanabe, "Lasing characteristics of GaInAsP-InP strained quantum-well microdisk injection lasers with diameter of 2-10 $\mu \mathrm{m}$," IEEE Photon. Technol. Lett., vol. 9, no. 7, pp. 878-880, Jul. 1997.

[3] M. Fujita, R. Ushigome, and T. Baba, "Continuous wave lasing in gainasp microdisk injection laser with threshold current of $40 \mu \mathrm{A}$," Electron. Lett., vol. 36, no. 9, pp. 790-791, Apr. 2000.

[4] J. Van Campenhout, P. Rojo-Romeo, D. Van Thourhout, C. Seassal, P. Regreny, L. D. Cioccio, J. M. Fedeli, C. Lagahe, and R. Baets, "Electrically pumped InP-based microdisk lasers integrated with a nanophotonic silicon-on-insulator waveguide circuit," Opt. Express, vol. 15, no. 11, pp. 6744-6749, 2007.

[5] S. J. Choi, Z. Peng, Q. Yang, S. J. Choi, and P. D. Dapkus, "Eight-channel microdisk CW laser arrays vertically coupled to common output bus waveguides," IEEE Photon. Technol. Lett., vol. 16, no. 2, pp. 356-358, Feb. 2004.

[6] D. Taillert, F. Van Laere, M. Ayre, W. Bogaerts, D. Van Thourhout, P. Bienstman, and R. Baets, "Grating couplers for coupling between optical fibers and nanophotonic waveguides," Jpn. J. Appl. Phys., vol. 45, no. 8A, pp. 6071-6077, 2006.

[7] J. V. Campenhout, P. Rojo-Romeo, D. V. Thourhout, C. Seassal, P. Regreny, L. D. Cioccio, J. M. Fedeli, and R. Baets, "Design and optimization of electrically injected InP-based microdisk lasers integrated on and coupled to a SOI waveguide circuit," J. Lightw. Technol., vol. 26, no. 1, pp. 52-63, Jan. 1, 2008

[8] J. V. Campenhout, P. Rojo-Romeo, D. V. Thourhout, C. Seassal, P. Regreny, L. D. Cioccio, J. M. Fedeli, and R. Baets, "Thermal characterization of electrically injected thin-film InGaAsP microdisk lasers on Si," J. Lightw. Technol., vol. 25, no. 6, pp. 1543-1548, Jun. 2007.

[9] H. G. Park, S. H. Kim, S. H. Kwon, Y. G. Ju, J. K. Yang, J. H. Baek, S. B. Kim, and Y. H. Lee, "Electrically driven single-cell photonic crystal laser," Science, vol. 305, no. 5689, pp. 1444-1447, Sep. 3, 2004. 